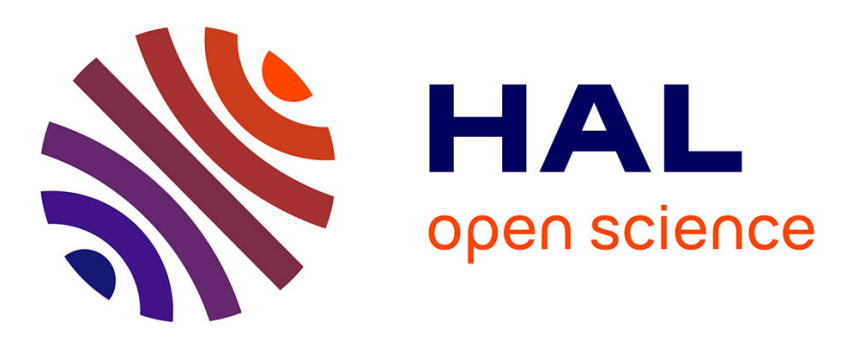

\title{
Pratiques et usages de l'information spécialisée chez les médecins spécialistes : place et rôle des structures documentaires
}

Céline Paganelli, Mounier Evelyne

\section{- To cite this version:}

Céline Paganelli, Mounier Evelyne. Pratiques et usages de l'information spécialisée chez les médecins spécialistes: place et rôle des structures documentaires. Tracer l'horizon informationnel du XXIe siècle: Frontières, passerelles et carrefours: 37ème congrès annuel de l'Association Canadienne des Sciences de l'Information, May 2009, Carleton University, Ottawa, Ontario., Canada. 19p. hal00651724

\section{HAL Id: hal-00651724 \\ https://hal.science/hal-00651724}

Submitted on 14 Dec 2011

HAL is a multi-disciplinary open access archive for the deposit and dissemination of scientific research documents, whether they are published or not. The documents may come from teaching and research institutions in France or abroad, or from public or private research centers.
L'archive ouverte pluridisciplinaire HAL, est destinée au dépôt et à la diffusion de documents scientifiques de niveau recherche, publiés ou non, émanant des établissements d'enseignement et de recherche français ou étrangers, des laboratoires publics ou privés. 
Evelyne Mounier

Céline Paganelli

Laboratoire Gresec

Université Stendhal-Grenoble

Etude réalisée dans le cadre des travaux du cluster 14 "Enjeux et représentations de la science, de la technologie et de leurs usages », cluster de recherche Rhône-Alpes.

\section{Pratiques et usages de l'information spécialisée chez les médecins spécialistes : place et rôle des structures documentaires}

Résumé. Variable selon les universités, et dispensée par les bibliothèques universitaires, la formation à l'information spécialisée est restée longtemps optionnelle pour tous les étudiants en médecine. La comparaison des usages de l'information spécialisée par les médecins spécialistes du secteur hospitalier et des spécialistes du secteur libéral, montre que ces derniers sont peu armés face aux mutations de leur domaine.

Abstract

\section{Introduction}

Les connaissances scientifiques et médicales évoluant rapidement, les médecins doivent réviser leurs connaissances et par là-même recourent régulièrement à l'information professionnelle ou scientifique de leur spécialité. La question de l'information des médecins est d'autant plus intéressante qu'ils peuvent être chercheurs et/ou simplement praticiens. De plus, en France, ils peuvent exercer soit en secteur public, notamment dans un centre hospitalier universitaire (CHU), soit en secteur privé dans un cabinet ou une clinique privée. Plusieurs questions se posent: quelles sont les pratiques informationnelles des médecins du secteur libéral ? Sont-elles identiques à celles de leurs collègues exerçant en CHU ? Cette étude porte sur les médecins spécialistes. Elle compare les pratiques 
informationnelles des médecins exerçant en CHU à celles des médecins exerçant en libéral et montre que, faute de formation suffisante à l'information scientifique et professionnelle pendant les études médicales, les spécialistes du secteur libéral sont moins bien armés que leurs collègues du CHU pour faire face aux mutations de leurs domaines de spécialité.

\section{Besoins et ressources en information chez les médecins}

En France les études médicales peuvent durer entre 9 et 13 ans selon le cas (généraliste/spécialiste), et l'on estime ( $\mathrm{CN}$ FMCH 2005) que 50\% des connaissances sont obsolètes dans un créneau de 7 ans. Ainsi, les médecins ne peuvent pas apporter des soins de qualité, tout au long de leur carrière, avec les seuls acquis de leur formation de départ. La mise jour des connaissances est un enjeu majeur pour eux.

Pour faire face à des besoins en information vastes et diversifiés, les médecins ont à leur disposition une offre d'information médicale diversifiée, immense et dont l'expansion sur le web n'est plus à démontrer. Par exemple, le catalogue CisMef produit par le CHU de Rouen recense plus de 43000 ressources francophones et intègre environ 50 nouvelles ressources chaque semaine. Toute tentative de prospection devient «à priori délicate» (Thirion, 1998), et cela d'autant plus que les ressources francophones ne représentent que $10 \%$ de la production mondiale médicale sur le Net et que l'anglais prédomine (CiSMef, 2009). Mais si les connaissances évoluent rapidement, d'autres approches comme la «médecine factuelle » et d'autres modes de formations (Apprentissage Par Problèmes, Apprentissage du Raisonnement Clinique) se développent également. Ainsi les médecins sont-ils amenés d'une part à assurer leur formation continue et d'autre part, à chercher de l'information d'autant plus qu'aujourd'hui 1 patient sur 5 (H.A.S, 2007) n'hésite pas à consulter les sites d'informations médicales sur le web et à poser à leur médecin de nombreuses questions (Gonod Boissin, 2005 ; Tietse, 2003 ; Croste, 2005).

\subsection{La formation à la recherche documentaire}

Depuis les années 90, les nouvelles technologies ayant bouleversé l'offre et les modes d'accès à l'information scientifique, toutes les bibliothèques universitaires et centres de 
documentation médicaux français ont organisé des formations à l'utilisation des ressources en ligne, destinées aux cursus médicaux. Barthet, Church et Dailland (Bathet, 2006) ont analysé l'offre proposée par 35 universités françaises et font plusieurs constats sur ces formations :

- Elles sont le plus souvent optionnelles et rarement incluses dans les cursus (Avala, 1999).

- Elles s'adressent à tous les étudiants, enseignants-chercheurs et praticiens (chefs de clinique, chefs de service), mais les internes et les thésards y sont les plus nombreux ; le nombre moyen de participants n'est que de deux à trois personnes par semaine. La plupart des étudiants ne voient pas l'utilité d'une telle formation.

- Elles sont assurées principalement par les bibliothécaires. La participation des médecins enseignants reste ponctuelle, la plupart n'y voyant pas grand intérêt parce que «les étudiants savent cliquer », qu'eux-mêmes ne sont pas formés et "qu'ils se débrouillent très bien » (Barthet, 2006).

Variable selon les universités, la formation est souvent axée sur l'utilisation de l'OPAC, les banques de données, la recherche sur internet, parfois sur des outils de veille (profils, alertes), souvent sur la gestion bibliographique. En général, elle comporte deux ou trois séances de travaux dirigés, autour de questions tirées des programmes. La plupart du temps, il n'y a pas de véritable évaluation.

Comme le souligne Eveillard (Eveillard, 2005), peu de choses ont été organisées en France pour apprendre aux étudiants à explorer, à trier et évaluer la quantité de données mises à disposition de tous. Pourtant, il se pourrait que cette situation évolue. En, effet depuis 2006 (circulaire $\mathrm{n}^{\circ}$ 2005-121 du 27 juillet 2005), en France, a été institué le certificat C2i commun à toutes les études supérieures et notamment le certificat $\mathrm{C} 2 \mathrm{i}$ de $2^{\text {ème }}$ année «métiers de la santé ». Lancé à titre expérimental pour 2006 et 2007, avec la participation de 11 établissements universitaires volontaires, le projet a été étendu à l'ensemble du pays. Cette formation est désormais obligatoire en France et elle doit respecter un référentiel (circulaire $n^{\circ} 2009-1006$ du 6 mars 2009) qui prévoit 4 modules dont un module «Recherche d'information en santé » structuré de la manière suivante : 
- les outils de codification de l'information en santé,

- la recherche d'information en santé,

- l'évaluation de l'information en santé sur internet,

- la mise en œuvre d'une veille documentaire professionnelle.

La formation $\mathrm{C} 2 \mathrm{i}{ }^{\circledR}$ niveau 2 «métiers de la santé » est accessible en formation initiale ou continue. Il suffit d'être titulaire du niveau1, ou de justifier des compétences requises par une procédure de validation des acquis. Le mode de formation préconisé est celui de l'autoformation basé sur la mutualisation des ressources sur un DVD $\mathrm{C} 2 \mathrm{i}{ }^{\circledR}$ niveau 2 «métiers de la santé » mis à disposition des universités. Les universités sont libres de compléter ce parcours par d'autres moyens : tutorat en ligne, enseignements dirigés. Les 4 modules prévus sont évalués par le biais de 2 épreuves et ils doivent être acquis pour que le $\mathrm{C} 2 \mathrm{i} \circledast$ niveau 2 «métiers de la santé » soit délivré.

Les représentants des bibliothèques n'ont pas été consultés pour la préparation de ce référentiel, mais la mise en œuvre d'un tel programme suppose le recours à des compétences variées : la participation des bibliothèques universitaires devrait s'imposer pour le module «Recherche d'informations » (Eveillard, 2005).

\subsection{La formation médicale continue (FMC)}

La formation médicale continue est une obligation spécifiée par le code de déontologie médicale $(\mathrm{CN}-\mathrm{FMCH} 2005)^{2}$ qui précis que «tout médecin doit entretenir et perfectionner ses connaissances; il doit prendre toutes dispositions nécessaires pour participer à des actions de formation continue ».

Pour valider l'obligation de FMC, chaque médecin doit obtenir un total de 250 crédits sur 5 ans. Tout d'abord, il devra se soumettre à des évaluations individuelles ou collectives de ses pratiques professionnelles, rendues obligatoires par le décret du 14 avril 2005 et qui devraient être confiées à des organismes de formation continue médicale agréés par la Haute Autorité de Santé et les organismes d'assurance maladie. Ensuite, chaque médecin est libre de choisir d'autres moyens parmi les possibilités suivantes : 
- formations personnelles: lectures de revues à comité de lecture, de livres, consultation de sites web médicaux agréés, etc.,

- formations en présentiel : séminaires, congrès, colloques, DU, DIU, EPU, staffs, séances de bibliographie,

- participation à des actions de recherche et formation : participation en tant que formateur FMI/FMC, publications,

- actions de santé publique : prévention, dépistage, participation à des réseaux de santé.

Mais ces dispositifs de formation continue n'envisagent pas que les compétences informationnelles puissent être enseignées et/ou entretenues au même titre que les connaissances médicales. Puisque les médecins sont libres de choisir leur mode formation continue, certains pourront peut-être décider de suivre le certificat $\mathrm{C} 2 \mathrm{i}$ «métiers de la santé ».

\section{Les pratiques informationnelles des médecins spécialistes}

En France, les médecins peuvent être enseignantschercheurs et/ou simplement praticiens. Les enseignants chercheurs en médecine et les médecins ayant des activités régulières de recherche, ont des pratiques informationnelles comparables à celles des autres scientifiques : en règle générale, ils lisent beaucoup (Tenopir 2003), privilégient les sources électroniques (DeGroote et Dorsch, 2001) (King et Montgomery, 2002) et les revues spécialisées en anglais, et utilisent les bibliothèques et les services documentaires comme fournisseurs d'accès unifié à des ressources électroniques (King, 2003). Mais cette communauté se démarque des autres communautés scientifiques : elle est très attachée aux circuits et aux sources connues et validées, ce qui évite perte de temps et vérifications (Tenopir 2003b)

S'agissant des médecins qui ne sont pas chercheurs, on remarque également qu'ils lisent plus que les physiciens ou les ingénieurs (Williamson 1989). Pour autant, leurs pratiques informationnelles sont-elles identiques à celles des confrères enseignants-chercheurs ? En quoi la formation à l'information scientifique et professionnelle éventuellement reçue lors des 
études universitaires peut-elle soutenir efficacement le besoin de mise à jour des connaissances, et les besoins des chercheurs ?

Cet article confronte les comportements informationnels de deux communautés de médecins spécialistes de la ville de Grenoble : des médecins exerçant au CHU et des médecins du secteur libéral. Les critères observés sont: l'effort de formation continue, les raisons induisant des recherches d'informations, la formation reçue en information scientifique et professionnelle, les conditions d'accès et les ressources effectivement utilisées. Les résultats concernant les spécialistes du CHU ayant déjà été présentés (Staii, 2008), seuls sont détaillés ici les résultats concernant la comparaison entre les 2 groupes de médecins.

\subsection{Méthodologie}

32 médecins ont été interrogés. Pour les médecins du $\mathrm{CHU}$, les 16 sujets enquêtés sont ceux qui ont répondu à la demande de rendez-vous émise par mail à l'ensemble des médecins spécialistes du CHU. Les spécialistes exerçant en libéral ont été contactés systématiquement par téléphone. Seuls 17 d'entre eux ont accepté d'être enquêtés. Un entretien a été rejeté parce qu'il ne s'est pas déroulé dans les mêmes conditions que les autres. Pour les 2 enquêtes, des entretiens semi-directifs ont été menés, structurés autour de thèmes majeurs: des questions d'ordre général, des questions sur les pratiques en matière de technologies de l'information et de la communication, des questions sur les pratiques informationnelles et la formation reçue dans ce domaine, et enfin des questions sur la formation continue. Pour les 2 groupes de médecins, la durée moyenne des entretiens a été de 45 à 60 minutes.

Tous les médecins interrogés disposent d'un équipement informatique et d'une connexion internet à domicile et travaillent dans un environnement technologique comparable : équipement du CHU pour les uns, cabinet privé informatisé avec accès haut débit à Internet pour les autres.

La répartition par âge montre une majorité de la tranche 31-40 ans pour les sujets du CHU et une majorité de la tranche 41-50 ans pour les médecins de ville. 
Une grande variété de spécialités médicales est représentée dans l'un et/ou l'autre des 2 groupes: cardiologie, angiologie, dermatologie, rhumatologie, radiologie, anesthésie-réanimation, chirurgie, gynécologie, médecine d'urgence, médecine du travail, gastro-entérologie, gériatrie.

Les médecins du secteur libéral qui exercent exclusivement en secteur privé (seuls en cabinet ou avec des associés ou en clinique.) représentent $58,90 \%$ des médecins interrogés. D'autres assurent également des consultations au CHU $(35,30 \%)$, ou assurent un service dans d'autres structures (18\%) tels que planning familial, centres de toxicologie, etc.

Outre les consultations, les médecins peuvent également avoir d'autres activités. Les spécialistes du secteur hospitalier sont largement occupés par leur carrière universitaire : recherche (75\%), activités d'enseignement (63\%). 56\% des médecins spécialisés du secteur libéral estiment ne pas avoir de temps disponible en dehors des consultations. Cependant, on constate que $31 \%$ des médecins du secteur libéral disent avoir une activité liée à un laboratoire pharmaceutique: par exemple comme consultant pour un laboratoire fabriquant des textiles, ou comme formateur pour des formations de médecins organisées par les laboratoires. En réalité, la plupart des médecins interagissent régulièrement avec les laboratoires pharmaceutiques: par exemple, ils participent comme formateurs ou comme formés à des formations commanditées par les laboratoires privés, reçoivent régulièrement les visiteurs médicaux.

\subsection{Résultats}

\subsubsection{La formation continue des médecins spécialistes}

Que ce soit en CHU ou en médecine de ville, la quasitotalité des médecins participe chaque année à un nombre important d'enseignements, réunions et/ou séminaires organisés par les CHU, les sociétés savantes, les associations de médecins, les réseaux de santé, associations de spécialistes et laboratoires pharmaceutiques. En moyenne, un médecin en suit au moins une vingtaine par an mais on remarque qu'en $\mathrm{CHU}$, les médecins 
sont aussi souvent formateurs que formés alors que les médecins de ville sont moins souvent formateurs. Parmi toutes les possibilités en formation continue, sont cités en priorité les enseignements post universitaires (EPU) et les congrès scientifiques.

Rares sont les spécialistes qui ne participent à aucun colloque scientifique de leur spécialité. Les spécialistes assistent à environ 2 à 5 colloques par an, ce qui représente une charge financière importante, surtout pour les médecins du secteur libéral qui doivent financer entièrement leur participation. C'est en CHU que l'on assiste à un plus grand nombre de colloques : les médecins y participent souvent en tant que chercheurs communiquants ou en tant que modérateurs. Il faut remarquer que certains médecins hospitaliers considèrent que la participation aux colloques ne relève pas de la formation continue mais de l'activité scientifique courante.

Un autre moyen de formation, également reconnu par la réglementation de la formation continue, est l'inscription dans des formations diplômantes. Les spécialistes du CHU tendent à privilégier les filières de recherche $(50 \%$ d'inscriptions en Master Recherche, en thèse et préparation d'HDR contre 13\% en DU), alors que les spécialistes du secteur libéral privilégient des formations appliquées à leur domaine et, s'ils sont moins nombreux à s'inscrire dans des formations universitaires (19\% d'inscriptions en DU), ils tendent à répéter ces certifications d'une année sur l'autre.

\subsubsection{Les raisons induisant des recherches d'informations}

Les situations citées comme amenant à chercher de l'information sont variées: activité clinique, recherche, enseignement, mise à jour des connaissances, réponses aux collègues, aux patients etc., Tous les médecins ont l'occasion de rechercher des informations pour fournir des réponses aux patients. C'est le cas par exemple quand des patients sont «allés sur internet et se sont fait peur ». Le médecin a alors souvent besoin d'images, pour illustrer l'évolution probable de leur cas particulier. 
En revanche, les deux populations observées se distinguent sur plusieurs points :

- Pour les spécialistes du CHU, ce sont des activités de recherche scientifique qui déclenchent principalement une recherche d'information. Cette forte activité de recherche d'information est liée naturellement à la conduite de projets de recherche, la préparation d'articles, la participation à des colloques mais aussi à des activités d'expertise et de reviewing ou évaluation de publications.

- La plupart des médecins hospitaliers de notre échantillon, assurent un service d'enseignement statutaire soit dans les cursus médicaux soit dans les écoles de soins (écoles de sagesfemmes, écoles d'infirmières etc.) ; la préparation de ces cours nécessite régulièrement des recherches d'informations, pour trouver des cours en ligne, des illustrations mais aussi pour donner des bibliographies indicatives aux étudiants.

- Pour les médecins du secteur libéral, ce sont les activités cliniques qui déclenchent le plus souvent les recherches d'information : devant une pathologie peu courante, on cherche à préciser un diagnostic, à choisir la meilleure indication thérapeutique. Il s'agit là de trouver des informations sur des cas précis. Certes, les médecins du CHU peuvent aussi rechercher de l'information pour préciser un diagnostic, déterminer la meilleure solution thérapeutique ou les meilleures pratiques à adopter mais il semblerait que ce soit surtout dans des secteurs polyvalents (comme les urgentistes), ou dans des secteurs où les données sont éparses comme la gériatrie par exemple.

Plusieurs types de besoins en informations apparaissent :

- des besoins en bibliographie (aussi exhaustives que possibles) : ce sont les chercheurs, les enseignants du CHU qui sont les plus forts demandeurs; en médecine libérale sont concernés ceux qui sont fortement impliqués dans des activités de consulting ou dans des formations en tant que formateurs ;

- des besoins d'informations très précises comme les pathologies rares, le choix de traitement, etc. ;

- des besoins ponctuels d'informations (vérification de geste technique par exemple, rafraichissement de connaissances, etc.). Ces deux derniers types de besoins peuvent concerner tous les médecins, mais sont directement liés à la clinique quotidienne : la recherche d'information prend du temps et le médecin n'a pas 
toujours la possibilité d'interrompre la consultation cours pour chercher des informations

\subsubsection{L'accès à l'information}

\subsubsection{Les ressources utilisées}

La première source d'information citée par l'ensemble des sujets est la bibliothèque personnelle composée de différents documents, disponibles à tout moment au bureau. On consulte régulièrement les traités et ouvrages de référence pour vérifier un point de détail, pour repréciser telle ou telle pathologie, revoir une procédure ou un geste technique. Le livre présente également tous les avantages des études synthétiques. L'ouvrage de référence est le document le plus utilisé par les médecins de ville. Dans la bibliothèque personnelle, on trouve également :

- des revues professionnelles (Quotidien du médecin, Prescrire) et des revues de spécialité qui sont lues régulièrement, sachant que la revue scientifique en anglais est la ressource privilégiée par les médecins chercheurs du CHU ;

- des documents fournis par les laboratoires pharmaceutiques et qui présentent des études récentes que les médecins de ville ne pourraient pas obtenir facilement. Ces documents sont envoyés gratuitement ou bien sont donnés par les visiteurs médicaux ;

- des documents récupérés dans le cadre des sessions de formation continue ou dans les congrès.

Toutes ces ressources sont majoritairement sur papier et sont peu organisées; ce qui ne gène pas nécessairement leur propriétaire qui «sait que c'est là en cas de besoin». En revanche, certains d'entre eux se constituent aussi des bibliothèques de favoris.

Les discussions entre confrères représentent aussi une source d'information importante pour les médecins en libéral : on peut discuter directement avec ses associés ou alors on téléphone si on a un doute, une hésitation. En ville, on utiliserait moins le contact par mail, moins rapide et moins direct que le téléphone.

Le recours aux bibliothèques n'est pas uniforme. S'agissant de la bibliothèque universitaire, dès la thèse terminée la grande majorité des médecins ne s'y déplace plus ou très peu. Les médecins du CHU préfèrent recourir aux services de la 
bibliothèque du service. Les spécialistes du secteur libéral (13\%) utilisent peu de telles ressources, à moins qu'ils assurent des vacations au $\mathrm{CHU}$ ou dans un organisme qui possède un service documentaire.

L'utilisation des ressources en ligne largement répandue pour les médecins chercheurs du CHU, tend à se généraliser pour les spécialistes du secteur libéral. Il est évident que la plupart des sujets interrogés peuvent faire des recherches par mots clé sur Google. Pubmed constitue la ressource principale (94\%) pour les médecins chercheurs du CHU, alors que $69 \%$ des médecins de ville ne l'utilisent pas du tout soit parce qu'ils n'ont pas besoin de faire de bibliographie, soit parce qu'ils ne savent plus l'interroger, soit encore parce que c'est l'obtention du document original qui pose problème. D'autres ressources scientifiques reconnues sont également citées par les médecins chercheurs du CHU : National Institute for Occupational Safety and Health (NIOSH), Cochrane Library ou the IsiWeb Of Science, Scholar Google, sociétés savantes, banques de données spécialisées.

Les spécialistes du secteur libéral mentionnent plutôt des sites de sociétés savantes, d'associations professionnelles, le Vidal en ligne, l'Encyclopédie médico-chirurgicale en ligne. Ignorés par les médecins de ville, le portail Gael de la bibliothèque universitaire est cité par la moitié des médecins du CHU qui utilisent donc les services de la bibliothèque pour accéder aux revues scientifiques en texte intégral, mais aussi pour commander des documents par le prêt entre bibliothèques (PEB).

Parmi les sites de référence, les deux groupes mentionnent le site du CHU de Rouen qui constitue une première orientation dans la profusion des ressources, des sites professionnels spécialisés, des sociétés savantes. La plupart de ces sites proposent des listes de diffusion auxquelles les médecins peuvent s'inscrire, ce qui leur permet de recevoir parfois des alertes scientifiques, mais aussi des informations diverses telles que des dates de congrès ou des informations d'actualité médicale.

\subsubsection{La formation à la recherche d'information}


Les médecins interrogés ne font pas de différence entre la formation à utiliser Google, qui n'existait pas pendant le cursus universitaire des plus âgés, et la formation à la méthodologie de la recherche bibliographique. Très généralement, les médecins des deux groupes se souviennent vaguement avoir reçu une sorte d'initiation à Medline au moment de leur thèse : "pendant la thèse, on avait un truc à la fac ... Medline, où je trouvais ce qu'il fallait pour mon sujet ». $\mathrm{Ce}$ qui ressort de l'ensemble des entretiens, c'est que la formation à la recherche d'information reçue pendant leurs études est presque toujours informelle, entre collègues :

«... il me semble qu’il y avait des choses proposées à la fac mais rien d'obligatoire pour qu'on nous montre comment rechercher une information. Mais en gros, en internat il y a des internes un peu plus âgés, qui sont là depuis un certain temps et qui nous montraient comment faire "viens je vais te montrer comment chercher dans Medline », ou la secrétaire, de la bibliothèque de l'internat qui nous initiait et puis après on se débrouillait». Rares sont ceux qui ont bénéficié d'une assistance à la bibliothèque universitaire, mais ceux-là ont apprécié l'aide des bibliothécaires : «On était aidé quand on avait une recherche à faire, elle nous disait quels mots taper. »

Aussi, alors que l'ensemble des médecins du CHU estime être suffisamment formés à la recherche d'information, les médecins de ville s'estiment peu performants : "oui, j'ai eu une formation mais je suis nulle quand même. Ca me permet juste de me débrouiller en consultation»

Seuls, $25 \%$ des médecins de ville interrogés ont bénéficié d'une formation, hors cursus universitaire. L'un d'eux a bénéficié d'une véritable formation à l'INIST : "C'était par une société qui s'occupait de veille, c'était sur Paris. Mais c'était quand j'étais dans l'industrie parce qu'une partie de mon activité consistait aussi à faire des recherches. Je me souviens oui j'étais allé à l'INIST faire une formation générale sur la veille. Par exemple j'ai appris à me servir des opérateurs, des choses comme ça. »; certains ont suivi une formation organisée par un laboratoire privé : «Un labo nous a offert cela (les labos Leo). C'est un médecin informaticien qui nous a appris à chercher des photos numériques, des informations. On était par groupes de 
10. Il est venu 2-3 fois à Grenoble»; d'autres ont reçu une telle formation dans le cadre d'un EPU: «On a participé, il y a quelques années à une formation par des médecins des centres de santé, qui faisaient des EPU. ».

Rares sont ceux qui, en dehors du circuit du CHU, sauraient comment obtenir les documents originaux. Un seul sujet estime maîtriser l'ensemble de la démarche depuis la recherche dans les bases de données bibliographiques jusqu'aux circuits d'obtention des documents : «(...) après je me débrouille pour trouver l'article. Soit je le fais commander, quand il a un rapport direct avec les industriels (...) Soit je vais le commander à la bibliothèque universitaire. Ou je le retrouve parfois sur des revues, enfin y a des photocopies au CHU dans les services qui relèvent de ma spécialité, je peux y aller aussi. (...) je fais partie d'un réseau de soins sur la phlébite et qui fait une veille bibliographique donc je peux m'adresser aussi à eux pour pouvoir obtenir des infos sur la phlébite si j'en ai besoin.. »

Le manque de formation à la recherche d'information rejaillit sur les pratiques. Ainsi $87 \%$ des médecins de ville estiment qu'il leur manque de la formation, de l'information et du temps pour rechercher de l'information, ce qui les amène à se montrer prudents vis-à-vis des informations médicales trouvées sur internet: "moi je l'utilise, je ne suis pas une pro donc je vais que sur ce que connais»; "on n'a pas la certitude que les informations soient toujours exactes donc on va toujours sur les mêmes sites ».

\section{Discussion}

Les médecins ayant accepté de participer à cette étude restent peu nombreux malgré les relances effectuées : manque de temps? inquiétudes? incompréhensions? Le caractère exploratoire de cette étude doit inciter à la prudence. En effet, ceux qui ont accepté sont probablement ceux qui sont déjà sensibilisés à cette problématique. Néanmoins, quelques constantes marquées apparaissent. Tout d'abord, pour les deux populations, on retrouve le besoin d'accéder à une information médicale validée mais aussi l'importance des discussions entre collègues, le recours aux personnes ressources pour obtenir 
directement l'information souhaitée ou pour être orienté. Tous assurent une veille scientifique et lisent régulièrement les principales revues de leur spécialité. Mais les chercheurs privilégient l'article scientifique en anglais, alors que les autres préfèrent le livre et les publications en langue française.

Pour les deux groupes, la formation continue représente un investissement important (en temps, en volume, en charge financière) mais dans le secteur public, la formation continue n'est pas dissociable de l'activité du chercheur. Dans le secteur libéral, où il est difficile d'accéder aux ressources documentaires de l'université, la formation continue, les colloques et séminaires représentent une source d'information capitale.

On constate pour les deux groupes, que la «formation» en recherche d'information reçue lors des études conditionne fortement le recours aux ressources en ligne pendant la vie professionnelle. Dans la mesure où l'on suit rarement les formations dispensées par la bibliothèque universitaire, on est «formés » entre collègues au moment de la thèse ; par la suite, les médecins tendent à reproduire ce qu'on leur a montré : puisqu'ils n'ont pas reçu de formation et qu'ils arrivent à trouver des documents, ils estiment souvent que leurs étudiants se débrouilleront aussi.

Par ailleurs, la recherche d'information est souvent assimilée de manière restrictive à la bibliographie et à l'exploitation des bases de données bibliographiques. Une telle conception gène peu le chercheur hospitalier tenu par la nécessité de publier, mais favorisé par l'appartenance à une équipe, l'accès aux réseaux documentaires de l'hôpital et aux services de la bibliothèque universitaire. En revanche, en secteur libéral, les médecins spécialistes ont peu d'occasions de faire des bibliographies et recherchent principalement des informations ponctuelles, des précisions, des rappels, des documents permettant de répondre à des questions émanant des patients. Ils cherchent davantage des informations précises, peut-être synthétiques, sans doute multimédia.

En secteur libéral, ceux qui font le plus de recherches d'informations sont ceux qui ont bénéficié de vraies formations. 
Mais, dans la majorité des cas, ces médecins manquent de la formation indispensable à la recherche d'information efficace sur le net et ils connaissent mal les ressources à leur disposition (portails universitaires, services offerts par les bibliothèques et possibilités d'accès, ressources publiques, etc). Qui plus est, ils ont peu de temps à consacrer aux recherches d'informations, et voudraient pouvoir lire davantage; on comprend ici l'importance des contacts avec les visiteurs médicaux, qui amènent informations et discussions (certes à but commercial), mais qui attirent aussi l'attention du médecin sur les évolutions les plus récentes des produits ou des traitements.

\section{Conclusions}

Le faible nombre de médecins interrogés doit susciter des interprétations prudentes. Néanmoins, les pratiques informationnelles observées chez des médecins spécialistes illustrent une situation préoccupante :

- D'un côté, des médecins spécialistes, dont la formation d'origine n'a pas intégré de formation à l'information scientifique et qui se doivent de faire face aux mutations rapides des connaissances. La culture informationnelle, au sens de « maîtrise de l'information » (Serres, 2007), des médecins est largement modelée par les usages et pratiques transmises lors de leurs études, par leurs aînés à 1'hôpital. Parce qu'ils sont extérieurs au cadre et aux ressources du CHU, les médecins du secteur libéral se sentent souvent isolés et démunis.

- De l'autre, des bibliothèques qui participent à «la mise en ordre de tout ce savoir disponible» (Thyrion, 1999), mais dont le rôle de formation est mal reconnu dans les cursus de santé mais aussi dont la mission de médiation semble s'amenuiser inexorablement du fait des facilités d'accès en ligne.

- Enfin, et entre ces deux acteurs, des sociétés commerciales qui investissent le créneau de publication scientifique, participent largement aux manifestations scientifiques, aux formations diverses en santé, à l'information commerciale, à la formation l'information professionnelle de santé.

C'est aux pouvoirs publics qu'il revient de mettre en place des moyens suffisants pour soutenir une politique de formation à la 
maîtrise de l'information scientifique et professionnelle à destination des personnels de santé, dans laquelle les bibliothèques peuvent trouver leur place à condition d'une collaboration avec les médecins enseignants et d'un ciblage sur les caractéristiques de la médecine factuelle ( $\mathrm{Li}, 2008)$.

\section{Références}

Alava, S. 1999. Médiations et métier d'étudiant ». Bulletin des bibliothèques de France. 1999, n¹, p. 8-15.

Barthet E., Amélie Cheurch, Françoise Dailland. 2006. Le lien discipline et formation à la maîtrise de l'information l'exemple de la médecine. Lyon: Enssib, mémoire de recherche, juin 2006, 84p.

$\mathrm{C} 2 \mathrm{i}$ certificat informatique et Internet « Métiers de la santé » (en ligne). Consulté le 14/03/2009. Disponible sur : http://c2i.education.fr/C2i2sante/index.ht

CISMef, 2009. CISMef : pourquoi, comment. (en ligne) Consulté le 8 avril 2009. Disponible sur: http://www.churouen.fr/cismef/cismef.html\#5

CN-FMC, 2005. L'obligation de formation médicale continue : historique, état des lieux et perspectives. Rapport du conseil national de la FMC des praticiens hospitaliers, 2005. Ressource (en ligne) Consulté le 29 novembre 2006. Disponible sur : http://www.sante.gouv.fr/htm/dossiers/obligation_fmc/accueil.ht $\underline{\mathrm{m}}$.

Croste, E. 2005. Utilisation de l'Internet dans le cadre de l'exercice professionnel. Enquête auprès de 300 médecins généralistes de l'Aquitaine. Thèse de médecine Bordeaux, 2005. (en ligne) Consulté le 7 décembre 2008. Disponible sur: 
http://www.medicalistes.org/spip/IMG/pdf/Internet-Medecine-

Generale.pdf.

DeGroote SL, Dorsch JL. 2001. Online Journals : Impact on Print Journal Usage. Bull Med Libr. Assoc. 89(4), p. 372-378, 2001.

Documentation française. 2007. L'information des médecins généralistes sur le médicament. Rapport de la Documentation Française, Paris, Inspection générale des affaires sociales, 2007. Eveillard, 2005. La Toile impose une formation initiale à la recherche documentaire. La Revue du Praticien en Médecine Générale, 21 novembre 2005,1319-1320.

Gonod-Boissin F. 2005. L'usage de l'information numérique en médecine générale : étude exploratoire en Rhône-Alpes. Thèse de doctorat en Sciences de l'information et de la communication, Université Lyon 1, 2007.

H.A.S, 2007. Haute Autorité de la Santé. Internet et santé, la certification : un moyen d'améliorer la qualité des sites dédiés à la santé. Communiqué de Presse du 27 novembre 2007. (en ligne) Consulté le 2 avril 2009 Disponible sur : http://www.hassante.fr/portail/jcms/c_607479/internet-et-sante-la-certificationun-moyen-dameliorer-la-qualite-des-sites-dedies-a-la-sante King DW, Montgomery CH 2002. After migration to an electronic journal collection: Impact on faculty and doctoral students. D-Lib Magazine, 8-12., 2002. (en ligne) consulté le 17.12.2008. Disponible sur

http://www.dlib.org//dlib/december02/king/12king.html, 
King DW, Boyce P, Montgomery CH, and Tenopir C 2003. Library economic metrics: Examples of the comparison of electronic and print journal collections and collection services. Library Trends. Vol. 51, No. 3, 2003.

Li Ping, Lin Wu. 2008. Are health sciences librarians taking the evidence-based medicine challenge ? $36^{\text {th }}$ annual conference CAIS-ACSI : LIS interacting with others disciplines. Vancouver, June 5-7, 2008

Serres A. 2007. Questions autour de la culture informationnelle. Canadian journal of information and library science / Revue canadienne des sciences de l'information et de la bibliothéconomie, vol. 31, ${ }^{\circ} 1$, mars 2007

Staii A, Balicco L, Bertier M, Clavier V, Mounier E et Paganelli C. 2008. Les pratiques informationnelles des médecins dans les centres hospitaliers universitaires : au croisement de la logique scientifique et de la culture professionnelle. Revue canadienne des sciences de l'information et de bibliothéconomie, Volume $30, \mathrm{n}^{\circ}$ 1/2, mars-juin 2008, pp. 57-69

Tenopir C., King D.W., and Bush A. 2003. Medical faculty's use of print and electronic journals: changes over time and comparison with other scientists. Journal of the Medical Library Association, 2003 (en ligne) consulté le 17.12.2008, Disponible sur

http://web.utk.edu/ tenopir/eprints/tenopir_jmla_article_042203 .pdf,)

Tenopir C ,King DW, and Bush A. 2003b. Medical faculty's use of print and electronic journals: changes over time and 
comparison with other scientists. Journal of the Medical Library Association, 2003 (en ligne) Consulté le 17/12/2008, Disponible sur

http://web.utk.edu/ tenopir/eprints/tenopir_jmla_article_042203 .pdf

Tietse S. 2003. Internet et capitalisation des connaissances en médecine: construction de la valeur d'usage des outils de l'Internet par les médecins hospitalo-universitaires. Thèse de doctorat, Université Claude Bernard, Lyon, 2003.

Williamson JW, German PS, Weiss R., Skinner EA, Bowes F. 1989. Health Science Information management and Continuing Education of Physicians: A Survey of US Primary Care Practitioners and Their Opinion Leaders, Annuals of Internal Medicine, 1989, 110(2), p. 151-160.

Remerciements : les auteurs remercient le docteur Guy-Noël Jacquet (chirurgie-ophtalmologie à Dax) et le docteur Brigitte Didier-Roberto (dermatologie à Grenoble) pour leurs conseils. 